\title{
AC Impedance-based Online State-of-charge Estimation for Li-ion Batteries
}

\author{
Shing-Lih $\mathrm{Wu},{ }^{1}$ Hung-Cheng Chen, ${ }^{2^{*}}$ and Ming-Yang Tsai ${ }^{2}$ \\ ${ }^{1}$ National Taitung Junior College, No. 911, Jhengci N. Rd., Taitung 95045, Taiwan \\ ${ }^{2}$ National Chin-Yi University of Technology, No. 57, Sec. 2, Chungshan Rd., Taiping Dist., Taichung 41170, Taiwan
}

(Received Nevember 11, 2017; accepted December 19, 2017)

Keywords: state-of-charge, AC impedance, online estimation, linear regression

The accurate estimation of state-of-charge (SOC) is one of the most important core functions of a battery management system (BMS), which provides the essential information needed for battery management, monitoring, and status analysis. This paper presents an online SOC estimation method based on AC impedance. A circuit for sensing the voltage and current of the battery is also proposed. The sensed values can be utilized to calculate the AC impedance and then accurately estimate the SOC of a Li-ion battery. First, a $1 \mathrm{kHz}$ sinusoidal ripple is injected into an $18650 \mathrm{Li}$-ion battery and the $\mathrm{AC}$ impedance values are measured from 0 to $100 \%$ charging status in 10\% increments. The correlations between SOC and AC impedance are then determined by linear regression. In terms of practical application, we simply use the sensing circuit to measure the battery $\mathrm{AC}$ voltage and current and calculate the $\mathrm{AC}$ impedance. This AC impedance allows a fairly accurate estimation of the battery SOC. To validate the accuracy of the proposed method, a power analyzer was used to actually measure SOC. The average error in estimating SOC is within $4 \%$. This encouraging result shows that the proposed method can provide a simple and practical solution for online estimation of the SOC of Li-ion batteries.

\section{Introduction}

Because traditional cars and trucks powered by fossil fuel account for most of the global greenhouse gas emissions, the electric vehicle industry is seeing rapid development. Consumer electronics is also booming thanks to the rapid advances in electronics technology. The power that drives electric cars and consumer electronics products mostly comes from secondary (rechargeable) batteries, most of which are Li-ion devices. ${ }^{(1-3)}$ However, the accurate estimation of the state of charge (SOC) of batteries is crucial to the battery management system (BMS). ${ }^{(4)}$ Currently, methods used for measuring battery SOC include Coulomb counting, open-circuit voltage, ampere-hour, internal resistance, and Kalman filtering.

Of these, Coulomb counting is the simplest method and the easiest to implement. It starts with the measurement of battery capacity. The battery SOC is then calculated from the battery output current and time. However, the process takes too long to achieve fast measurement of 
battery SOC. ${ }^{(5)}$ In the open-circuit voltage method, SOC estimation is achieved by comparing open-circuit voltages. However, the load on the circuit has to be disconnected and the battery must return to electrochemical equilibrium before this can be done, so the SOC cannot be obtained in real time. ${ }^{(6)}$ The ampere-hour method calculates battery SOC by multiplying the current flowing in or out of the battery by the time of flow. However, in addition to current, battery capacity is affected by temperature and other factors and this may lead to errors in calculation. ${ }^{(7)}$ In the internal resistance method, SOC estimation is carried out by measuring the internal resistance of the battery, but rather expensive instrumentation is needed for this. The method cannot be used when the battery is under load. ${ }^{(8)}$ The battery SOC measurement methods mentioned thus far herein require that the battery be isolated from the system, which is both inconvenient and likely to cause errors. ${ }^{(9)}$

This paper presents a method of sensing the battery voltage and current circuit through the measured value to calculate the battery AC impedance and estimate the battery SOC by a method in which a $1 \mathrm{kHz}$ sinusoidal ripple is fed into a $18650 \mathrm{Li}$-ion battery. The AC impedance is measured between 0 and $100 \%$ SOC. The relationship between the SOC of the battery and the $\mathrm{AC}$ impedance is then determined by linear regression. The error rate of the SOC estimation using this method is less than $4 \%$.

\section{AC Impedance Analysis of Li-ion Batteries}

The rapid development of the portable electronics industry has led to the development of Li-ion batteries, which are now the most widely used portable power source. Li-ion batteries exhibit various properties which depend on the anode materials used, and different materials are used for different applications. Figure 1 shows the equivalent circuit of a Liion battery. In this figure, $E_{b}$ is the ideal battery voltage; $L_{e}$ denotes the electrode inductance between the anode and cathode; $R_{O}$ represents the ohmic resistance created by the electrode metals, electrolyte concentration and welding points; $C_{D L}$ refers to the capacitance formed by the double-layer effect, where the valence charges on the electrode surface attract ions with opposite charges; $R_{C T}$ is the charge transfer resistance, which affects the speed a charge moves between electrode surfaces; and $Z_{W}$ represents the Warburg impedance, created by ion diffusion when the chemical reaction is strongly affected by reactants approaching or products escaping

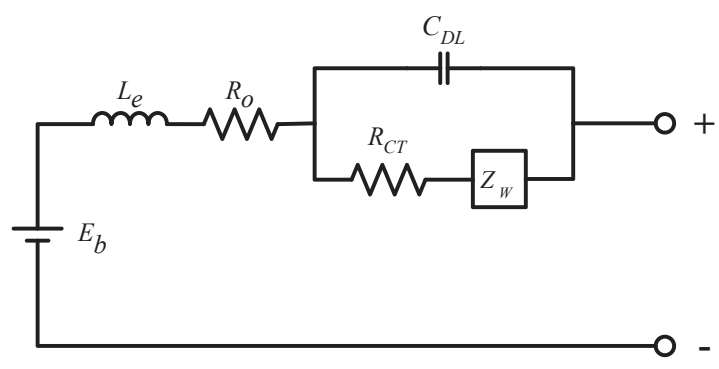

Fig. 1. Equivalent circuit of Li-ion battery. 
from electrode surfaces. In addition, the AC impedance of the battery changes under different charging frequencies. In the high-frequency region $(>1 \mathrm{kHz})$, a passivation layer is formed. In the medium- and low-frequency ranges $(<1 \mathrm{~Hz}$ and $1 \mathrm{~Hz}-1 \mathrm{kHz}$, respectively), the battery is in the charge transfer and electron transition states, respectively.

In Fig. 1, the Warburg impedance occurs only in the low-frequency range and parasitic inductance is prominent in the high-frequency range. To simplify the equivalent circuit inside the battery, the small signal frequency which was injected by the sensing circuit in this study was $1 \mathrm{kHz}$ so that the battery Warburg impedance and the parasitic inductance associated with the low- and high-frequency ranges can be neglected. The simplified equivalent circuit is shown in Fig. 2. ${ }^{(8-10)}$

Sensing the battery voltage with the current inside the circuit was accomplished by feeding a sine wave and charging voltage to a voltage/current converter circuit, as shown in Fig. 3. The $V_{S}(t)$ driven by operational amplifier (OPA) to control the switch of metal-oxide-semiconductor field-effect transistor (MOSFET) and then converted into an AC current through $R_{S}$ resistance. According to the analysis described, the AC impedance of the battery can be calculated by measuring the battery ripple voltage and current. The input signal is provided by a sine wave generator circuit with a DC voltage added, as shown in Eq. (1). The waveform is shown in Fig. 4. It can be found that the sine wave level increases with the DC voltage, so the sine wave can be completely input to the switch and will not be cut off. The output signal of the voltage/current converter runs through the resistor $R_{S}$ and generates a current, which is shown in Eq. (2). The voltage signal across the battery is derived from this current passing through the battery, shown in Eq. (3). The waveform is shown in Fig. 5.

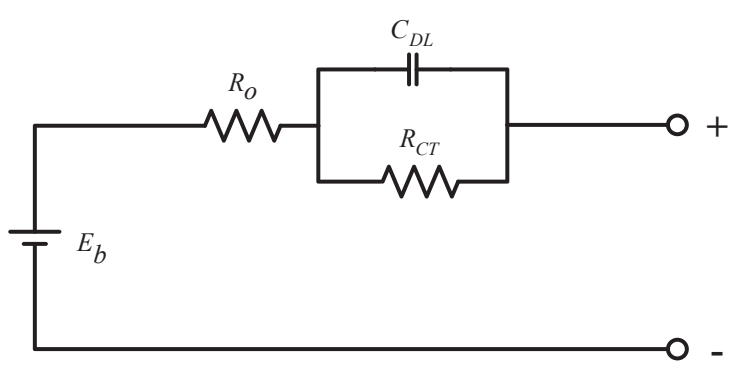

Fig. 2. Simplified equivalent circuit of Li-ion battery.

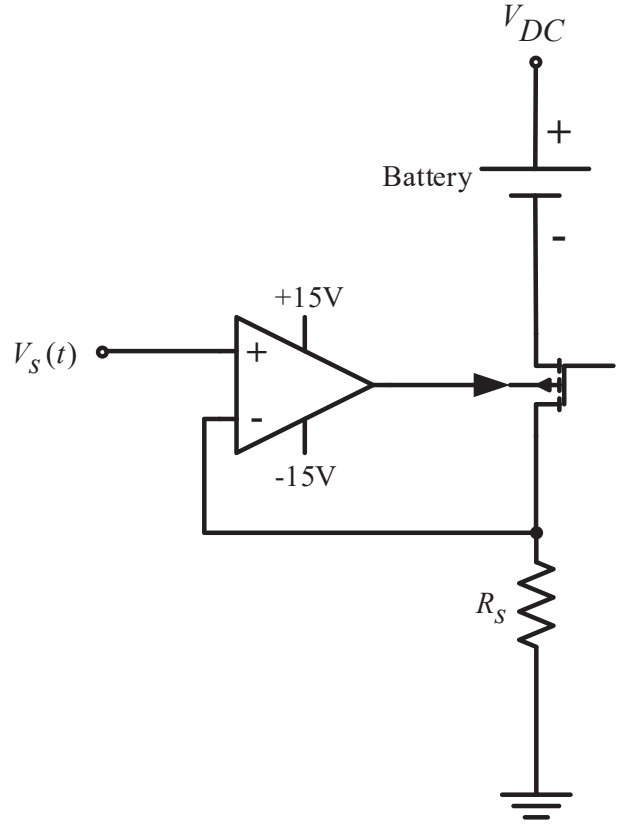

Fig. 3. Voltage/current converter circuit. 


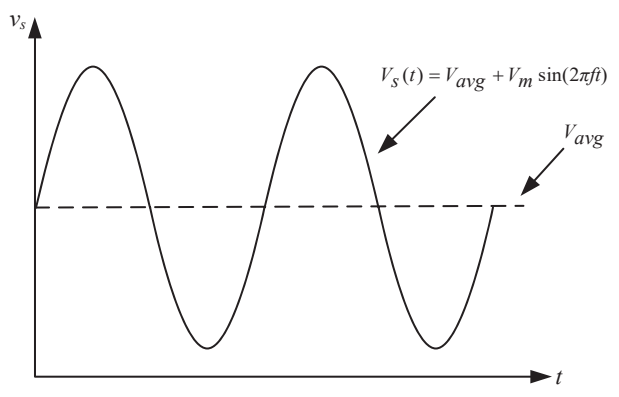

Fig. 4. Sine wave with a DC waveform.

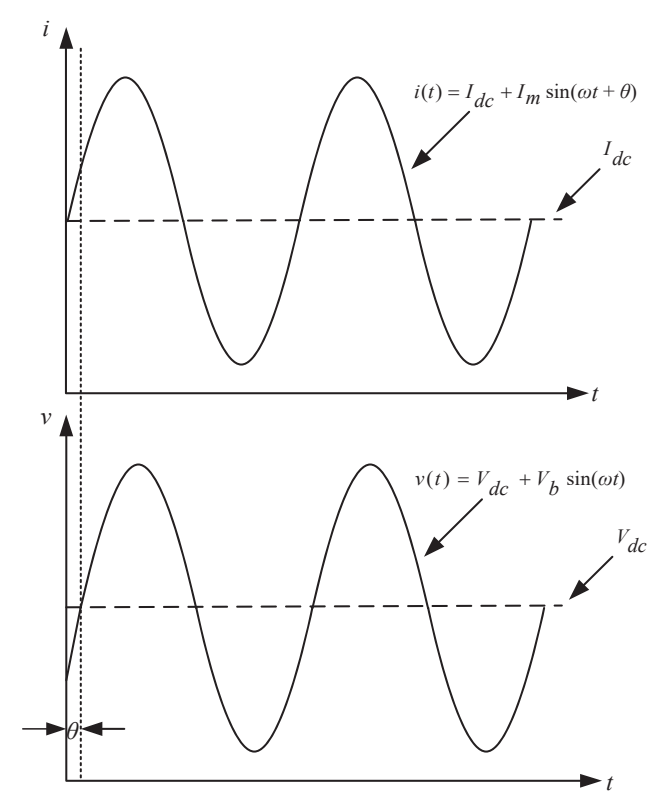

Fig. 5. Waveforms of AC current and battery voltage.

$$
v_{s}(t)=V_{a v g}+V_{m} \sin (2 \pi f t),
$$

where $V_{a v g}$ is the average DC voltage and $V_{m}$ is the peak value of the sine wave signal.

$$
i(t)=\frac{v_{s}(t)}{R_{s}}=I_{d c}+I_{m} \sin (\omega t+\theta),
$$

where $I_{d c}$ is the average current and $I_{m}$ is the value of the peak sinusoidal current.

$$
v(t)=V_{d c}+V_{b} \sin (\omega t),
$$

where $V_{d c}$ is the DC voltage and $V_{b}$ the value of the peak battery sinusoidal voltage.

As shown in Eq. (4), the battery impedance is obtained by dividing Eqs. (2) by (3).

$$
\bar{Z}=\frac{V_{m}}{I_{m}} \angle-\theta
$$

Lastly, the impedance $\bar{Z}$ in Eq. (4) is transformed into the complex form shown in Eq. (5).

$$
\bar{Z}=Z^{\prime}-j Z^{\prime \prime}=|Z| \angle-\theta
$$

where $Z^{\prime}$ is the real part of the impedance (the resistance component); $Z^{\prime \prime}$, the imaginary part (the 
reactance component); $|Z|$, the maximum value of the $\mathrm{AC}$ impedance equation; and $\theta$ the phase angle of AC impedance, the phase difference between battery voltage and current.

Figure 6 shows the internal circuit of the battery voltage and current sensing circuit that consists of an adder, a voltage/current converter, and bandpass filter.

As shown in Fig. 7, the sine wave is generated using the sine wave generating circuit. The elements $R_{1}, R_{2}, C_{1}$, and $C_{2}$ are designed to produce a $1 \mathrm{kHz}$ sine wave. The design formula is shown in Eq. (6). It should also be noted that $R_{4}$ divided $R_{3}$ must be greater than or equal to 2 so that it will produce a shock. The output of the sine wave easily becomes unstable, so the output oscillation voltage of full-wave rectifier must go through the regulator circuit. The OPA2 output is a negative rectified voltage. The voltage is then passed through the $1 \mathrm{~S} 1588, R_{7}$, and $C_{4}$ filter to obtain a negative DC voltage, which is almost equal to the peak output of the oscillation output. This negative voltage is applied to the G pole of the junction FET (JFET) to control the resistance between the D-S poles.

$$
f_{o}=\frac{1}{2 \pi \sqrt{R_{1} R_{2} C_{1} C_{2}}}
$$

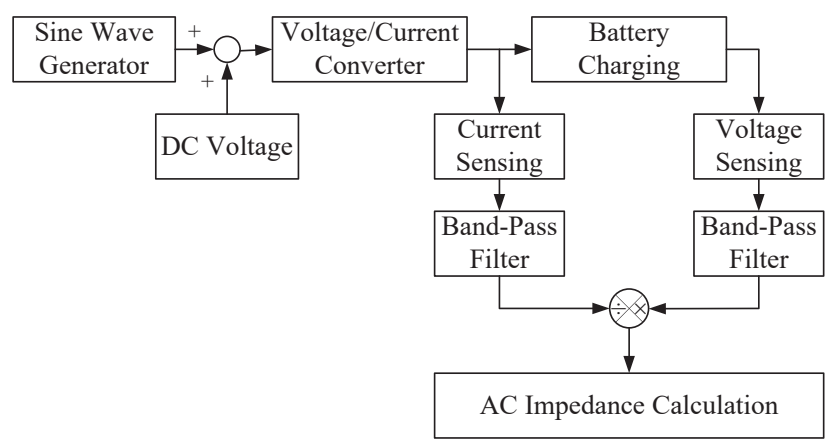

Fig. 6. Block diagram of battery voltage and current sensing and AC impedance calculation.

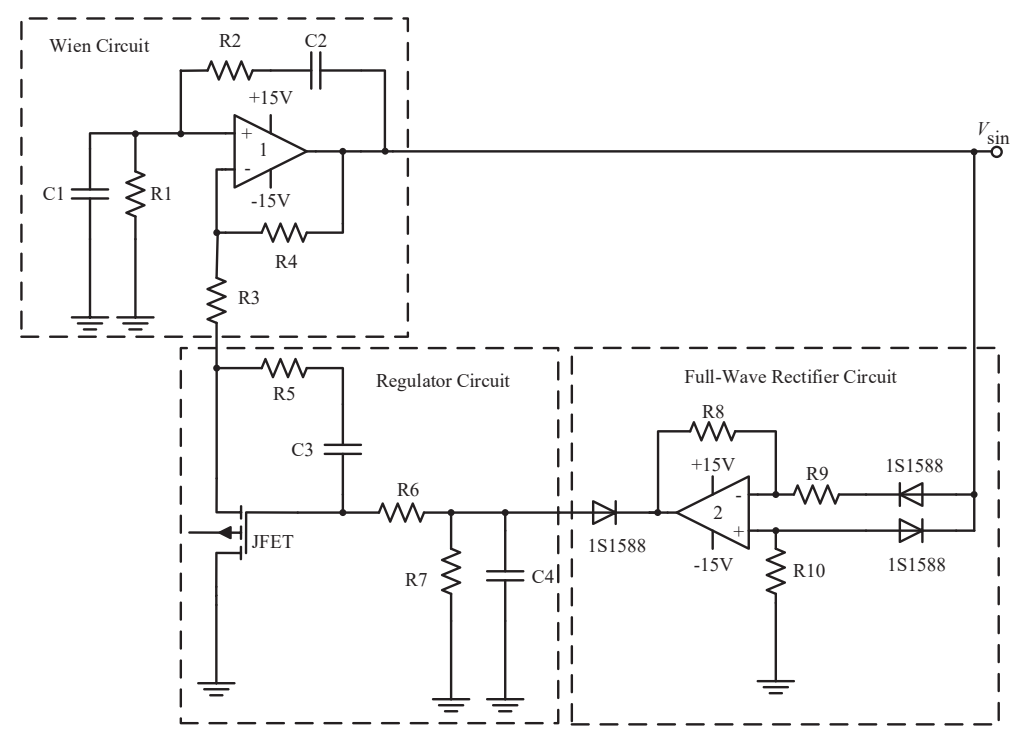

Fig. 7. Sine wave generation circuit. 
where $f_{o}$ is the frequency of the sine wave for the Wien bridge oscillator, and $R_{1}=R_{2}$ and $C_{1}=$ $C_{2}$.

To allow the sine wave to pass through the voltage of the voltage/current converter, we need to use a non-inverting adder to boost sine wave level, as shown in Fig. 8. The output voltage of the adder is expressed in Eq. (7).

$$
v_{s}(t)=\left(1+\frac{R_{14}}{R_{13}}\right) \frac{V_{\text {sin }}+V_{\text {avg }}}{2},
$$

where $R_{11}=R_{12}=R_{13}=R_{14}$, so that the output $v_{s}(t)$ can be equal to $V_{\sin }$ with the addition of $V_{\text {avg. }}$.

Utilizing the voltage/current converter the sine wave is injected into the battery and the battery sine wave voltage can then be obtained. Since the ripple voltage is too small to measure, the study uses the band-pass technique to filter high-frequency noise and battery DC voltage, so that the battery voltage and current sensing circuit produce better measurements. As shown in Fig. 9, a band-pass filter is designed to determine a resonance frequency of $1 \mathrm{kHz}$ and a bandwidth of $200 \mathrm{~Hz}$, and then to calculate $R_{15}, R_{16}$, and $R_{17}$ using Eqs. (8)-(11).

$$
f_{r}=B Q
$$

where $f_{r}$ is the resonant frequency, $B$ is the frequency bandwidth, and $Q$ is the quality factor.

$$
R_{15}=\frac{1}{2 \pi A B C}
$$

where $A$ is the voltage gain, and $C_{5}=C_{6}=C$ is the designed capacitance.

$$
\begin{gathered}
R_{16}=\frac{R_{1}}{2 Q^{2}-1} \\
R_{17}=\frac{1}{\pi B C}
\end{gathered}
$$

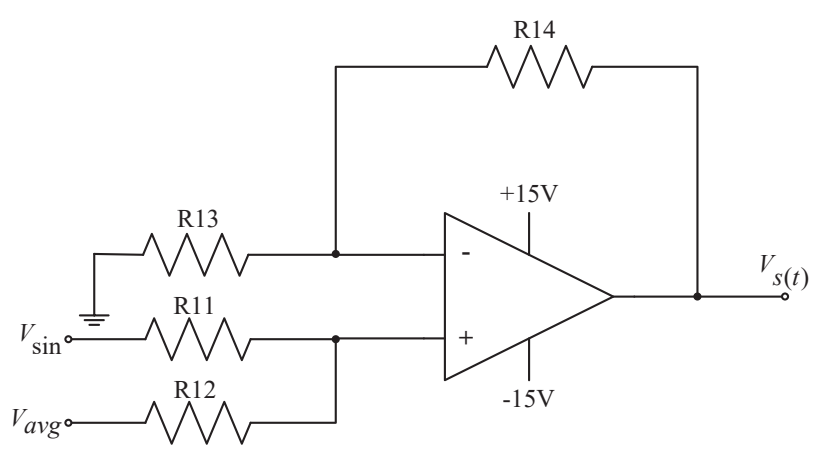

Fig. 8. Non-reverse adder.

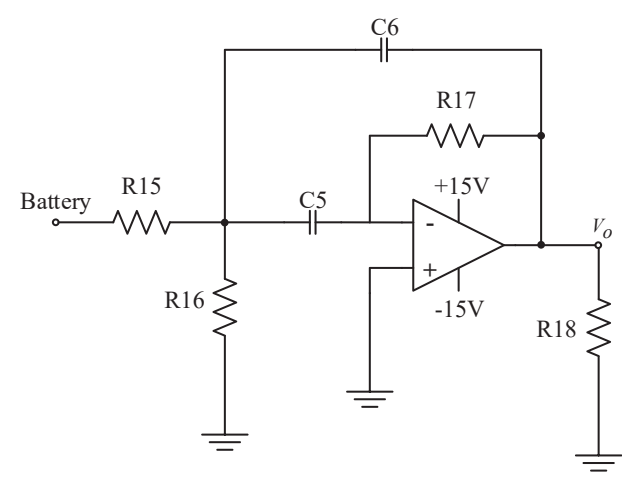

Fig. 9. Band-pass filter. 


\section{Linear-regression-based SOC Estimation}

The AC battery impedance measurement procedure works as follows: A sine wave with a DC offset is fed into the voltage/current converter, and the current is then injected into the battery. The magnitude of the current used to charge the battery can be adjusted through the variable resistor in the voltage/current converter. The $\mathrm{AC}$ impedance is then calculated by measuring the $\mathrm{AC}$ voltage and $\mathrm{AC}$ current across the battery. The measurements were taken at 11 points: $0,10,20,30,40,50,60,70,80,90$, and $100 \%$ SOC, and the correlation was then determined by linear regression. A simple linear regression model is shown in Eq. (12), and the sample linear regression equation was obtained using the least-squares method as shown in Eq. (13). This was then used to calculate the equation governing the relationship between the AC impedance of the battery and the SOC. Once this is done, the battery SOC can be obtained at any time by simply measuring the $\mathrm{AC}$ impedance and substituting it into the equation.

$$
Y_{i}=\beta_{0}+\beta_{1} X_{i}+\varepsilon_{i}
$$

where $Y_{i}$ represents the $i$-th observed value; $X_{i}$, the independent variable corresponding to the $i$-th observed value; $\beta_{0}$, the intercept; $\beta_{1}$, the slope; and $\varepsilon_{i}$, the random error.

$$
\hat{Y}_{i}=b_{0}+b_{1} X_{i},
$$

where $\hat{Y}_{i}$ represents $Y$ the average of estimates for specific $X_{i}$, and $b_{0}$ and $b_{1}$ represent the unbiased estimates of $\beta_{0}$ and $\beta_{1}$, respectively.

The experiment was conducted as follows. First, a sine wave from the AC generator circuit was added to the charging voltage. The signal was fed into the voltage/current converter so that a ripple voltage was added to the DC current. To avoid a short circuit and overcome the difficulty of measuring the battery voltage, which is greater than the ripple voltage, the ripple voltage and charging current were fed into an INA128 IC and a band-pass filter before being measured. The AC impedance of the battery was measured at 11 SOC points, and linear regression was used to determine the relationship between AC impedance and SOC. Using this method, the SOC of the battery can be determined at any time, simply by measuring the AC impedance. A flow chart of the experiment is shown in Fig. 10.

\section{Experimental Results and Discussions}

ICR-18650M Li-ion batteries were used in the experiment, and their specifications are shown in Table 1.

The method presented in this paper was used to test the relationship between the AC impedance of the battery and the SOC over a range of 0 to $100 \%$ SOC. A charging signal of 1 $\mathrm{kHz}$ was used. The AC impedance for 0, 50, and 100\% SOC is shown in Figs. 11-13.

Using the voltage and current measured by oscilloscope, after a simple calculation, we can 


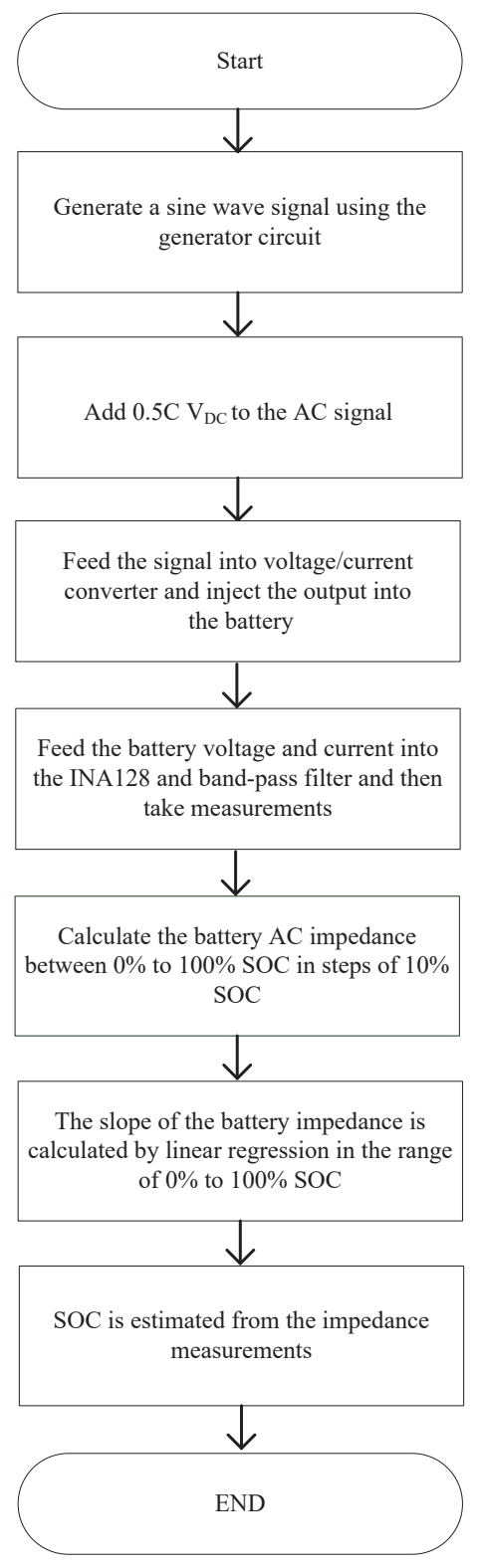

Fig. 10. Experiment flow chart.

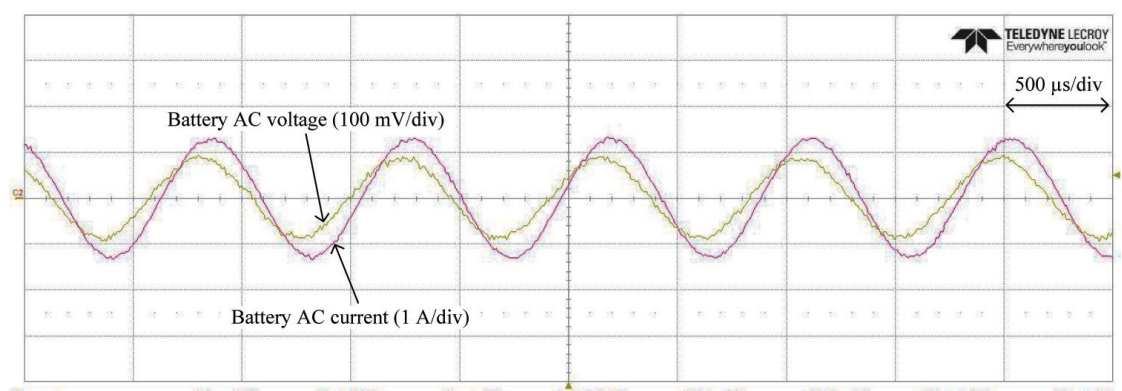

Fig. 11. (Color online) Battery AC voltage and charging current at $0 \%$ SOC.

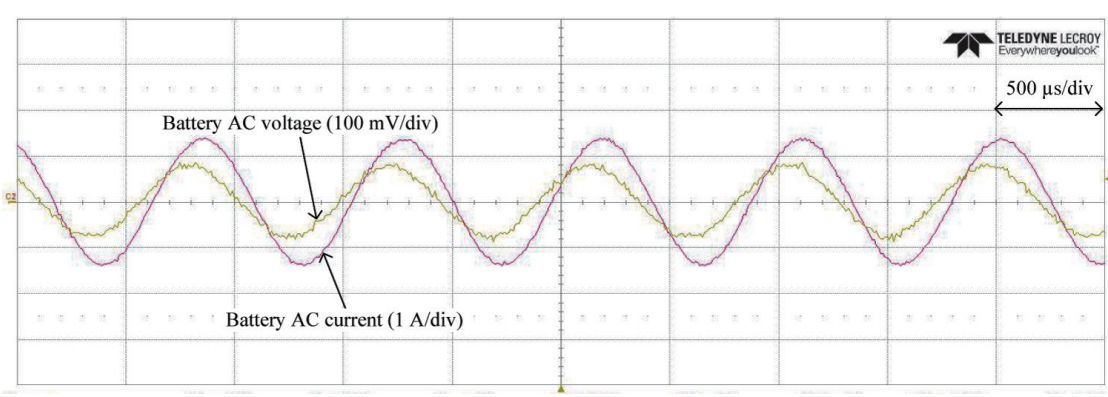

Fig. 12. (Color online) Battery AC voltage and charging current at $50 \% \mathrm{SOC}$.

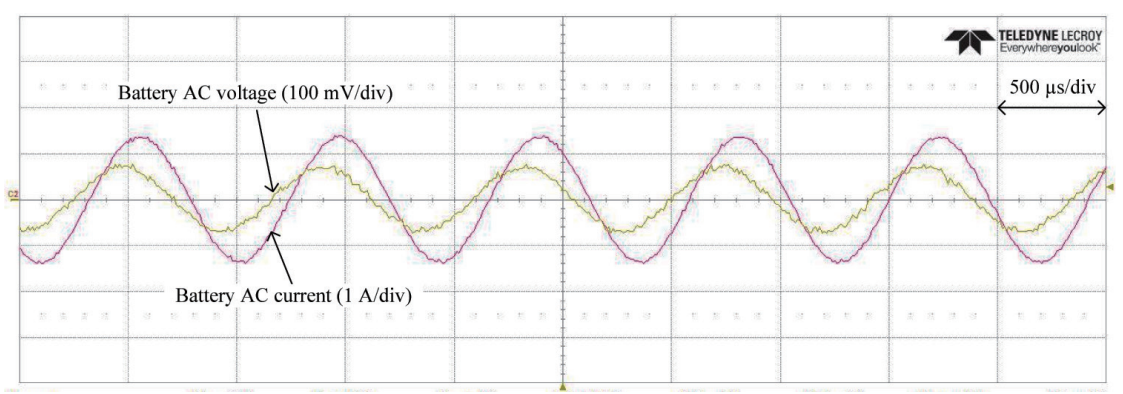

Fig. 13. (Color online) Battery AC voltage and charging current at $100 \%$ SOC.

Table 1

Specifications of ICR-18650M Li-ion battery.

\begin{tabular}{lc}
\hline Item & Specifications \\
\hline Rated capacity & $2800 \mathrm{mAh}$ \\
Rated voltage & $3.7 \mathrm{~V}$ \\
Maximum charging voltage & $4.2 \mathrm{~V}$ \\
Minimum discharge voltage & $3 \mathrm{~V}$ \\
Maximum charging current & $2.8 \mathrm{~A}$ \\
Maximum discharge current & $5 \mathrm{~A}$ \\
Internal resistance AC $(1 \mathrm{kHz})$ & $70 \mathrm{~m} \Omega$ \\
\hline
\end{tabular}


get the size of the battery $\mathrm{AC}$ impedance. The $\mathrm{AC}$ impedance between 0 and $100 \% \mathrm{SOC}$ is shown in Fig. 14.

It can be seen that the AC impedance of the battery changes because of the different SOC, and it was found that the larger the SOC of the battery, the smaller the impedance. The use of this battery impedance trend can be used to estimate the actual battery SOC.

The equation governing the relationship between battery AC impedance and the SOC, as shown in Eq. (14), is determined by linear regression from SOC data obtained at 11 points between 0 and $100 \%$. The estimated AC impedance at the points is obtained by substituting the data into the equation. The comparison between the $\mathrm{AC}$ impedance, estimated and measured, is shown in Table 2. The average error was $1.567 \%$ and the highest individual error was less than $4 \%$. Figure 15 shows the $\mathrm{AC}$ impedance, estimated by linear regression, in the range of 0 to $100 \%$ SOC.

$$
\hat{Y}_{i}=67.911809-0.132418 X_{i}
$$

where $\hat{Y}_{i}$ is the AC impedance in $\mathrm{m} \Omega$ and $X_{i}$ is the battery SOC, the ratio of the remaining battery capacity over total capacity as a percentage.

To verify the relationship between battery SOC and AC impedance, an impedance analyzer was used to measure the $\mathrm{AC}$ impedance of five batteries at 11 points in the range of 0 to $100 \%$

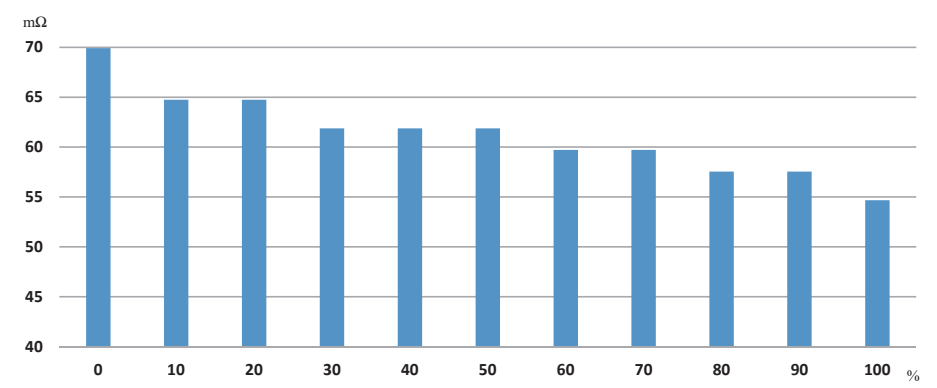

Fig. 14. (Color online) Battery AC impedance between 0 and $100 \%$ SOC.

Table 2

Accuracy analysis of the AC impedance estimated by linear regression approach.

\begin{tabular}{rccc}
\hline $\begin{array}{l}\text { Battery SOC } \\
(\%)\end{array}$ & $\begin{array}{c}\text { AC impedance by } \\
\text { measurement }(\mathrm{m} \Omega)\end{array}$ & $\begin{array}{c}\text { AC impedance by } \\
\text { linear regression }(\mathrm{m} \Omega)\end{array}$ & $\begin{array}{c}\text { Error } \\
(\%)\end{array}$ \\
\hline 0 & 69.92 & 67.91 & 2.874 \\
10 & 64.74 & 66.58 & 2.763 \\
20 & 64.74 & 65.26 & 0.796 \\
30 & 61.87 & 63.93 & 3.222 \\
40 & 61.87 & 62.61 & 1.181 \\
50 & 61.87 & 61.29 & 0.946 \\
60 & 59.71 & 59.96 & 0.416 \\
70 & 59.71 & 58.64 & 1.824 \\
80 & 57.55 & 57.31 & 0.418 \\
90 & 57.55 & 55.99 & 2.786 \\
100 & 54.67 & 54.66 & 0.018 \\
\hline
\end{tabular}




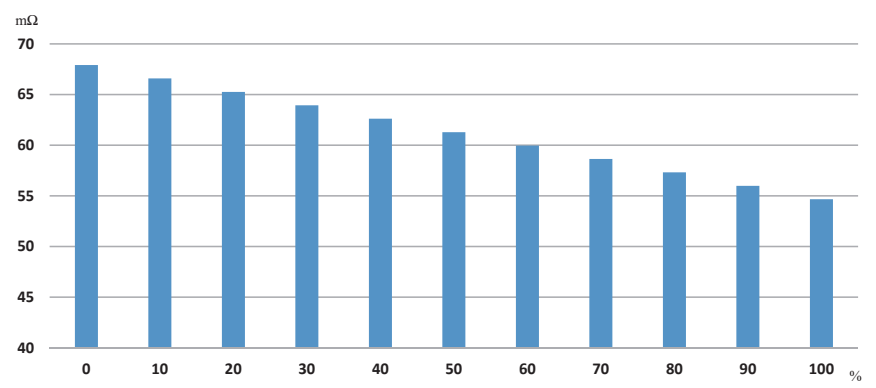

Fig. 15. (Color online) Battery SOC estimated from battery AC impedance by linear regression.

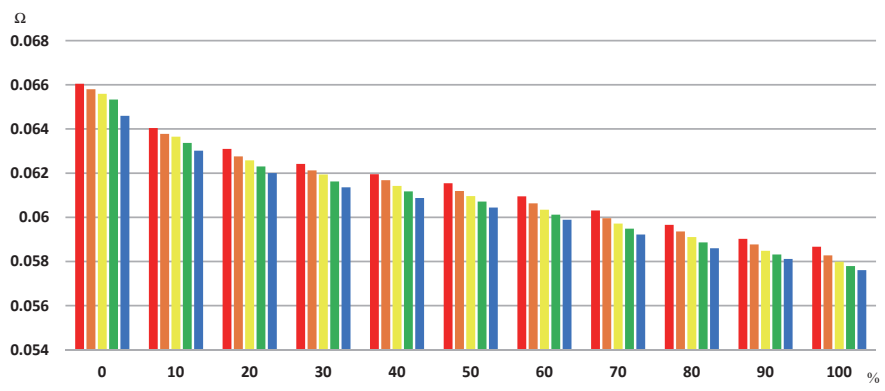

Fig. 16. (Color online) AC impedance of five Li-ion battery samples over the range of 0 to $100 \%$ SOC.

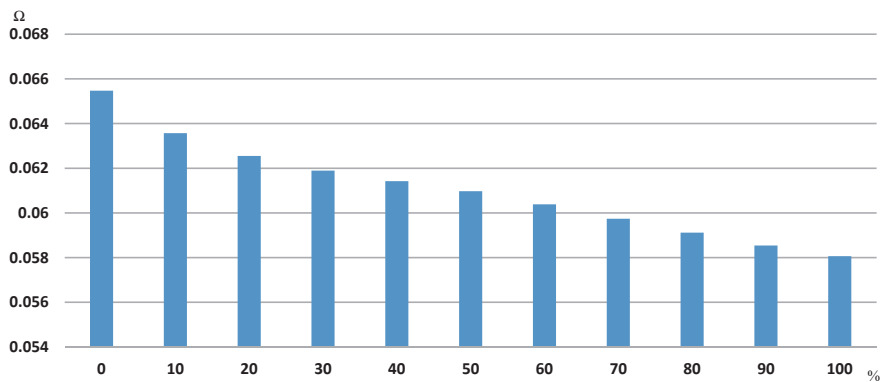

Fig. 17. (Color online) Average AC impedance of five Li-ion battery samples over the range of 0 to $100 \%$ SOC.

SOC with a charging signal of $1 \mathrm{kHz}$ frequency. The relationship between the AC impedance and SOC of the samples is shown in Fig. 16. The average AC impedance of the five batteries confirms that the AC impedance becomes smaller as the state of charge grows, as shown in Fig. 17 , where the $x$-axis represents SOC and the $y$-axis AC impedance of the batteries.

\section{Conclusions}

An online method for the estimation of SOC of Li-ion batteries based on the battery AC impedance has been presented. The method can be used when the battery is under load. The $\mathrm{AC}$ impedance of the battery can be quickly calculated with battery voltage and current sensing circuits measuring the voltage and ripple current. Fast SOC estimation can be achieved by 
substituting the $\mathrm{AC}$ impedance into a linear equation governing the relationship between the battery SOC and AC impedance. Unlike the open-circuit methods, there is no need to isolate the load and wait for the battery to return to electrochemical equilibrium before the measurements are taken. No expensive instruments, such as those used for measurement of the internal resistance, are required. The $1.567 \%$ average error rate and largest single error of less than $4 \%$ make this method useful and reliable.

\section{Acknowledgments}

This research was partially supported by the Ministry of Science and Technology of Taiwan, under Grant MOST 106-2221-E-167-017.

\section{References}

1 K. Hu, V. Sivaraman, B. G. Luxan, and A. Rahman: IEEE Sens. J. 16 (2016) 1448.

S. Jeong, Y. J. Jang, and D. Kum: IEEE Trans. Power Electron. 30 (2015) 6368.

3 C. J. Govar and J. A. Banner: IEEE Aerosp. Electron. Syst. Mag. 18 (2013) 17.

4 R. Ahmed, M. E. Sayed, I. Arasaratnam, J. Tjong, and S. Habibi: IEEE J. Emerging Sel. Top. Power Electron. 2 (2014) 678.

5 A. H. Ranjbar, A. Banaei, A. Khoobroo, and B. Fahimi: IEEE Trans. Smart Grid 3 (2012) 360.

6 M. Coleman, C. K. Lee, C. Zhu, and W. G. Hurley: IEEE Trans. Ind. Electron. 54 (2007) 2550.

7 K. T. Lee, M. J. Dai, and C. C. Chuang: IEEE Trans. Ind. Electron. 65 (2018) 589.

8 Y. D. Lee, S. Y. Park, S. B. Han, K. Norregaard, L. Overgaard, and F. Elefsen: IEEE Trans. Ind. Appl. 51 (2015) 498.

9 Y. Ma, B. Li, G. Li, J. Zhang, and H. Chen: IEEE/CAA J. Autom. Sin. 4 (2017) 195.

10 L. R. Chen, S. L. Wu, and D. T. Shieh: IEEE Trans. Ind. Electron. 60 (2013) 88.

11 S. L. Wu, H. C. Chen, and S. R. Chou: Energies 3438 (2014) 11.

\section{About the Authors}

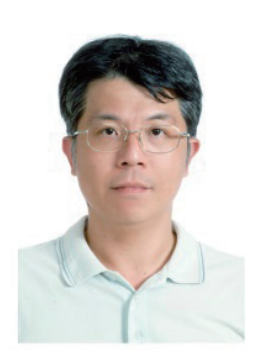

Shing-Lih Wu received his B.S. degree form National Formosa University, Yunlin, Taiwan, in 2003, his M.S. degree in the Department of Electrical Engineering, Feng-Chia University, Taichung, Taiwan, in 2005, and his Ph.D. degree in the Department of Electrical Engineering, National Changhua University of Education, Changhua, Taiwan, in 2010. He joined the faculty of the Department of Electrical Engineering, National Taitung Junior College, Taitung, in February 2014, where he is currently an Assistant Professor. His major research interests are power electronics, battery-powered circuit design, renewable energy, battery chargers, and control applications.

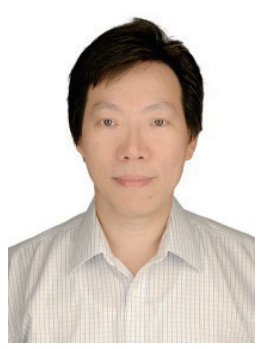

Hung-Cheng Chen received his M.S. and Ph.D. degrees, both in electrical engineering, from the National Taiwan University of Science and Technology in 1989 and 1993, respectively. In February 1993, he joined National Chin-Yi University of Technology, Taichung, Taiwan, as a faculty member. $\mathrm{He}$ is presently a Professor in the Department of Electrical Engineering. His major areas of research include micro-grids, smart grids, fault diagnosis, and partial discharge detection. 
Ming-Yang Tsai received his B.S. degree from National Penghu University of Science and Technology, Penghu, Taiwan, in 2015. He is currently working toward his M.S. degree in the Department of Electrical Engineering, National Chin-Yi University of Technology, Taichung, Taiwan. His major research interests are battery chargers and control applications. 